\title{
On the Infinite Products of Matrices
}

\author{
Yousry S. Hanna ${ }^{1}$, Samya F. Ragheb ${ }^{2}$ \\ ${ }^{1}$ National Research Institute of Astronomy and Geophysics, Helwan, Egypt \\ ${ }^{2}$ Faculty of Science, Helwan University, Helwan, Egypt \\ Email: yousry_hanna@yahoo.com
}

Received May 29, 2012; revised June 27, 2012; accepted July 5, 2012

\begin{abstract}
In different fields in space researches, Scientists are in need to deal with the product of matrices. In this paper, we develop conditions under which a product $\prod_{i=0}^{\infty} P_{i}$ of matrices chosen from a possibly infinite set of matrices $M=\left\{P_{j}, j \in J\right\}$ converges. There exists a vector norm such that all matrices in $M$ are no expansive with respect to this norm and also a subsequence $\left\{i_{k}\right\}_{k=0}^{\infty}$ of the sequence of nonnegative integers such that the corresponding sequence of operators $\left\{P_{i_{k}}\right\}_{k=0}^{\infty}$ converges to an operator which is paracontracting with respect to this norm. The continuity of the limit of the product of matrices as a function of the sequences $\left\{i_{k}\right\}_{k=0}^{\infty}$ is deduced. The results are applied to the convergence of inner-outer iteration schemes for solving singular consistent linear systems of equations, where the outer splitting is regular and the inner splitting is weak regular.
\end{abstract}

Keywords: Matrices; Infinite Products; Iteration

\section{Introduction}

Let the standard iterative method for solving the system of linear equations

$$
A x=b
$$

where $A \in R^{n, n}$ and $x, b$ are n-vectors [1], be induced by the splitting of $A$ into $A=T-Q$, where $T$ is a nonsingular matrix. Starting with an arbitrary vector $x_{o}$, the recurrence relation

$$
T x_{k+1}=Q x_{k}+b
$$

is used to compute a sequence of iterations whose limit should be the solution to Equation (1).

If $A$ is a nonsingular matrix, to obtain a good approximation to the solution of Equation (1), one need not to even solve the system (2) exactly for each $x_{k+1}$. For each $k \geq 1$, we solve the system (2) by iterations. Then split the matrix $\mathrm{T}$ into

$$
T=G-H
$$

where the matrix $G$ is invertible. Then, starting with $y_{o}:=z_{k}, t_{k}$ inner iterations

$$
y_{j}=G^{-1} H y_{j-1}+G^{-1} c, \quad c=Q z_{k}+b, j=1, \cdots, t_{k}
$$

are computed after which one resets $z_{k+1}=y_{t k}$. The entire inner-outer iteration process can then be expressed as follows [2-4]

$$
\begin{aligned}
z_{k+1}= & \left(G^{-1} H\right)^{t_{k}} z_{k}+\sum_{i=0}^{t_{k}-1}\left(G^{-1} H\right)^{i} G^{-1} b \\
= & \left\{\left(G^{-1} H\right)^{t_{k}}+\sum_{i=0}^{t_{k}-1}\left(G^{-1} H\right)^{i} G^{-1} Q\right\} z_{k} \\
& +\sum_{i=0}^{t_{k}-1}\left(G^{-1} H\right)^{i} G^{-1} b \\
= & P_{t_{k}} z_{k}+\sum_{i=0}^{t_{k}-1}\left(G^{-1} H\right)^{i} G^{-1} b,
\end{aligned}
$$

where

$$
P_{t_{k}}:=\left(G^{-1} H\right)^{t_{k}}+\sum_{i=0}^{t_{k}-1}\left(G^{-1} H\right)^{i} G^{-1} Q, \quad k=1,2, \cdots
$$

If the spectral radius of both $T^{-1} Q$ and $G^{-1} H$ are smaller than 1 so that the powers of both iteration matrices converge to zero, then for sufficiently large positive integer $t$ we have that if $t_{k} \geq t \forall k \geq 1$ [5], the sequence $\left\{z_{k}\right\}$ produced by the inner-outer iterations converges to the solution of Equation (1) from all initial vectors $z_{0}$. If $A$ and $T$ have a nonnegative inverse and both iteration matrices $T^{-1} Q$ and $G^{-1} H$ are nonnegative matrices, with the former induced by a regular splitting of $A$ and the latter induced by a weak regular splitting of $T$, then 
the sequence $\left\{z_{k}\right\}$ converges to the solution of Equation (1) whenever $t_{k} \geq t \forall k \geq 1$ with no restrictions on $t$ [4]. The process of inner-outer iterations can be represented by means of an iteration matrix at every stage, the spectral radius of such a matrix can no longer be less than 1. Furthermore, even if the spectral radius of the iteration matrix at each stage is 1 , this does not ensure the convergence of the inner-outer iteration process even if a fixed number of iterations are used between every two outer iterations [6]. If the number of inner iterations, between every two outer iterations, is allowed to vary, the problem is further compounded $[7,8]$. Here we shall examine some connections between the work here and problems of convergence of infinite products of matrices such as considered by [6].

If one is going to employ the inner-outer iteration scheme, then it is very reasonable that often between any two outer iterations only a relatively small number of inner iterations will be computed and only in rare cases much more inner iteration will be allowed. This effectively means that there is a number $n \geq 1$, such that infinitely often at most $n$ inner iterations will be carried out between any two outer ones. This implies that there exists an index $1 \leq n_{o} \leq n$ such that for an infinite subsequence $i_{k}$ of the positive integers, $t i_{k}=n_{o}$, infinitely often, $P_{t i_{k}}=P_{m_{0}}$. We shall prove that under certain convergence properties of $P_{m_{0}}$ such as $P_{m_{o}}$ is paracontracting with respect to a vector norm in respect of which all the $P_{i, s}$ are no expansive, the inner-outer iteration (5) for any initial vector $z_{o}$. This implies that the inner-outer iteration scheme is convergent when the system (1) is consistent.

Now let we have an infinite set of matrices

$M=\left\{P_{j}, j \in J\right\}$, and there exists a vector norm $\|\cdot\|$ on $\mathrm{C}^{\mathrm{n}}$ such that each matrix in $\mathrm{M}$ is no expansive with respect to $\|\cdot\|$. From $\mathrm{M}$ select an infinite sequence of matrices $\left\{P_{i}\right\}_{i=0}^{\infty}$. Then if $\left\{P_{i}\right\}_{i=0}^{\infty}$ contains a subsequence $\left\{P_{i_{k}}\right\}_{k=0}^{\infty}$ which converges to a matrix $H$ which is paracontracting with respect to $\|\cdot\|$ and such that the null space $N(I-H)$ is contained in the intersection of the null spaces $N(I-H) N\left(I-P_{j}\right), j \in J$, then

$\exists \lim _{i \rightarrow \infty} P_{i} P_{i-1} \cdots P_{o}$. Finally, let $D$ be the set of all sequences $(c)=\left\{c_{i}\right\}_{i=0}^{\infty}$ of integers such that each sequence $(c)$ contains an integer $k=k^{(c)}$ such that $c_{i}=k$ for infinitely many $i^{s}$. Then, according to Th. 3.1 if corresponding to the sequence $(c)$, the matrix $P_{k}$ is para- contracting, then

$$
\exists \lim _{i \rightarrow \infty} P_{c_{i}} \cdots P_{i_{o}}=: P^{(c)}
$$

We shall show that the function $f:(c) \rightarrow P^{(c)}$ is continuous.

\section{Preliminaries}

Let $E \in C^{n, n}$. We shall denote both of the null space and the range of $E$ by $N(E)$ and $R(E)$ respectively. Recall that the Jordan blocks of $E$ corresponding to 0 are 1 $\times 1$ if and only if

$$
N(E) \cap R(E)=\{0\}
$$

and

$$
N(E)+R(E)=C^{n},
$$

a situation which we shall write as

$$
N(E) \oplus R(E)=C^{n} .
$$

Recall further that according to [9] the powers of a matrix $E \in C^{n, n}$ converges if and only if

$$
N(I-E) \oplus R(I-E)=C^{n}
$$

and

$$
\gamma(E):=\max \{|\lambda|, \lambda \in \sigma(E), \lambda \neq 1\}<1,
$$

where $\sigma(\cdot)$ denotes the spectrum of a matrix.

For a vector $x \in R^{n}$ we shall write that $x \gg 0(x>0)(x \geq 0)$ if all the entries of $x$ are positive numbers. Also, let $\|\cdot\|$ enote a vector norm in. An $n \times n$ matrix $E$ is no expansive with respect to $\|\cdot\|$ if for all $x \in C^{n}$,

$$
\|E x\| \leq\|x\|
$$

$E$ is called paracontracting with respect to $\|\cdot\|$ if for all $x \in C^{n}$

$$
E x \neq x \Leftrightarrow\|E x\|<\|x\|
$$

We denote by $N(\|\cdot\|)$ the set of all matrices in $C^{n, n}$ which are paracontracting with respect to $\|\cdot\|$. Two examples of paracontracting matrices are as follows. For the Euclidian norm it is known that any Hermitian matrix whose eigenvalues lie in $(-1,1]$ is paracontracting. Suppose now that $E$ is an $n \times n$ positive matrix whose spectral radius is 1 and with a Perron vector $x \gg 0$. We claim that such a matrix is paracontracting with respect to $\|\cdot\|_{x}$, the monotonic vector norm induced by $x$. Let $y \in R^{n}$ be any vector satisfying $y \neq E y$ or, equivalently, not being a multiple of $x$. We know that

$$
\|y\|_{x}=\min \{\delta>0,-\delta x \leq y \leq \delta x\}
$$

By the positively of $\mathrm{E}$ and because $E x=x$, it follows that for any $\delta$ such that $-\delta x \leq y \leq \delta x,-\delta x \ll E y \ll \delta x$, so that $\|E y\|_{x}<\|y\|_{x}$.

The concept of paracontraction was introduced by [4] who showed that the product of any number of matrices in $N(\|\cdot\|)$ is again an element of $N(\|\cdot\|)$. Moreover, they used a result of [3] to show that the powers of any matrix $E \in N(\|\cdot\|)$ converge. Thus, in particular such 
matrix has the property that

$$
N(I-E) \oplus R(I-E)=C^{n, n} .
$$

Finally, recall that a splitting of $A$ into $A=T-Q$ is called regular if $T$ is nonsingular, $T^{-1} \geq 0$ and $Q \geq 0$. Regular splitting where introduced by [10], who showed that for a regular splitting, $\rho\left(T^{-1} Q\right)<1$ if and only if $\mathrm{A}$ is nonsingular and $A^{-1} \geq 0$. A splitting $A=T-Q$ is called weak regular if $T$ is nonsingular, $T^{-1} \geq 0$ and $T^{-1} Q \geq 0$. This concept was introduced by [11] who showed that, even allowing for this weakening of the assumption on regular splitting, $\rho\left(T^{-1} Q\right)<1$ if and only if $A$ is nonsingular and $A^{-1} \geq 0$. If $A=T-Q$ is a regular splitting of $A$, then

$$
\rho\left(T^{-1} Q\right) \leq 1
$$

and

$$
R\left(I-T^{-1} Q\right) \oplus N\left(I-T^{-1} Q\right)=R^{n}
$$

if and only if $A$ is range monotone [12], that is,

$$
[A x \geq 0 \text { and } x \in R(A)] \Rightarrow x \geq 0 .
$$

Moreover, they showed that if there exists a vector $x \gg 0$ such that $T^{-1} Q x \leq x$, then $\rho\left(T^{-1} Q\right) \leq 1$ and $R\left(I-T^{-1} Q\right) \oplus N\left(I-T^{-1} Q\right)=R^{n}$, and such a positive vector always exists if $A$ is a singular and irreducible M-matrix.

\section{Applications to Singular Systems}

As we mentioned before, if $A=T-Q$ is a regular splitting for $A \in R^{n, n}$ and $A$ is range monotone, then $\rho\left(T^{-1} Q\right) \leq 1$ and

$$
R\left(I-T^{-1} Q\right) \oplus N\left(I-T^{-1} Q\right)=R^{n} .
$$

Now, let $T=G-H$ is a weak regular splitting for $T$ and consider the inner-outer iteration process

$$
\begin{aligned}
z_{k+1}= & \left(G^{-1} H\right)^{T k} z_{k}+\sum_{j=0}^{T k-1}\left(G^{-1} H\right)^{j} G^{-1} C \\
= & \left\{\left(G^{-1} H\right)^{T k}+\sum_{j=0}^{T k-1}\left(G^{-1} H\right) G^{-1} Q\right\} z_{k} \\
& +\sum_{j=0}^{T k-1}\left(G^{-1} H\right)^{j} G^{-1} C \\
= & \mathrm{P}_{\mathrm{Tk}} z_{k}+\sum_{j=0}^{T k-1}\left(G^{-1} H\right)^{j} G^{-1} C
\end{aligned}
$$

where

$$
P_{i}=\left(G^{-1} H\right)^{i}+\sum_{j=0}^{i-1}\left(G^{-1} H\right)^{j} G^{-1} Q, \quad i=1,2, \cdots
$$

We observe at once that since $A=T-Q$ is a regular splitting for $A$ and $T=G-H$ is a weak regular split- ting for $T$, any of the inner-outer iteration operators $P_{i}, i \geq 1$, is a nonnegative matrix. Already Nichols in [3] essentially showed the following relation holds:

$$
I-P_{i}=\left(I-R^{i}\right)\left(I-T^{-1} Q\right),
$$

Suppose that the $\mathrm{n} \times \mathrm{n}$ coefficient matrix $A$ (assumed to be nonsingular) in the system (1) is monotone. For each $i \geq 1$, let $A=T_{i}-Q_{i}$ be a regular splitting of $A$ and $T_{i}=G_{i}-H_{i}$ be a weak regular splitting. Consider the inner-outer iteration process:

$$
z_{i+1}=P_{i, T i} Z_{i}+\sum_{i=0}^{T i-1}\left(G_{i}^{-1} H_{i}\right)^{i} G_{i}^{-1} Q_{i} b,
$$

where as, in the introduction, $T_{i} \geq 1$ and

$$
P_{i, T i}=\left(G_{i}^{-1} H_{i}\right)^{T i}+\sum_{j=0}^{T i-1}\left(G_{i}^{-1} H_{i}\right)^{j} G_{i}^{-1} Q_{i}
$$

If there are splitting $A=T-Q$ and $T=G-H$ such that for infinitely many is $T_{i}=T$ and $G_{i}=G$ simultaneously, then for any $z_{o} \in R^{n}$,

$$
\lim _{i \rightarrow \infty} z_{i}=A^{-1} b
$$

Now, suppose $A \in R^{n, n}$ is a range monotone and that $A=T-Q$ and $T=G-H$ are regular and weak regular splitting for A and $\mathrm{T}$ respectively, then $\rho\left(P_{i}\right) \leq 1$ and

$$
N\left(I-P_{i}\right) \oplus R\left(I-P_{i}\right)=R^{n}
$$

for all $i \geq 1 \quad[2,10,13]$.

Once again, suppose that $A=T-Q$ and $T=G-H$ are regular and weak regular splitting for $A$ and $T$ respectively. Note that the range monotone of $A$ was used only to deduce that $I-T^{-1} Q$ is an M-matrix of index at most 1. Another condition which ensures that $I-T^{-1} Q$ is an $M$-matrix of index at most 1 is that there exists a positive vector $x$ such that $A x \geq 0$ for then $T^{-1} Q x \leq x$. Furthermore, such a vector exists when $A$ is a singular and irreducible $M$-matrix. When $A$ is such an $M$-matrix, then, in fact, there exists a positive vector $x$ such that $A x=0$. But then also

$$
T^{-1} A x=x-T^{-1} Q x=0
$$

so that $x=T^{-1} Q x$, and hence

$$
P_{i} x=T^{-1} Q x+R^{i}\left(I-T^{-1} Q\right) x=T^{-1} Q x=x
$$

We can thus conclude that when $A$ is an irreducible $M$-matrix, not only the conclusions of the above result hold, but $P_{i} x=x$ so that $\left\|P_{i}\right\|_{x}=1$. Hence for each $i \geq 1, \quad P_{i}$ is no expansive with respect to the norm $\|\cdot\|_{x}$. We also see that

$$
0=G^{-1} A x=x-G^{-1} H x-G^{-1} Q x \leq x-G^{-1} H x=x-R x
$$

Now we know that $Q \geq 0$. Thus if either $G^{-1} Q x \gg 0$ 
or $G^{-1} H x \gg 0$, then it follows that $x \gg R x$ so that inductively,

$$
1>\|R\|_{X} \geq\|R\|_{X}^{2} \geq \cdots
$$

Let $V:=T^{-1} Q$. Then from the relation

$$
P_{i}-V=R^{i}(I-V)
$$

we see that, not only

$$
\lim _{i \rightarrow \infty} P_{i}=V,
$$

a fact that already follows from $\rho(R)<1$, but that the rate of convergence behaves as $\|R\|_{X}$.

Theorem: Let $M=\left\{P_{j}, j \in J\right\}$ be a set of matrices in $C^{n, n}$, let $\left\{P_{i}\right\}_{i=0}^{\infty}$ be a sequence of matrices chosen from $M$, and consider the iteration scheme

$$
x_{i+1}=P_{i} x_{i}, i=0,1,2, \cdots
$$

Suppose that all $P_{j} \in M$ are no expansive with respect to the same vector norm $\|$.$\| and there exists a subse-$ quence $\left\{P_{i k}\right\}_{k=0}^{\infty}$ of the sequence $\left\{P_{i}\right\}_{i=0}^{\infty}$ such that $\lim _{k \rightarrow \infty} P_{i k}=V$ where $V$ is a matrix with the following properties:

- $\mathrm{V}$ is paracontracting with respect to $\|\cdot\|$.

- $N(I-V) \subseteq \bigcap_{j \in J} N\left(I-P_{j}\right)$

Then for any $x_{o} \in C^{n}$ the sequence $x_{i+1}=P_{i} x_{i}$ is convergent and

$$
\lim _{i \rightarrow \infty} x_{i} \in N(I-V) \subseteq \bigcap_{j \in J} N\left(I-P_{j}\right) .
$$

The proof is given in [2].

From the above analysis and previous theorem we can now state the following result concerning the convergence of the inner-outer iteration process:

Theorem: Let $A \in R^{n, n}$ and suppose that $A=T-Q$ and $T=G-H$ are a regular splitting and a weak regular splitting for $A$ and $T$, respectively, and consider the inner-outer iteration process (7) for solving the consistent linear system $A x=b$. Suppose there exists a vector $x \gg 0$ such that $A x \geq 0$ and one of the following conditions is satisfied:

1) For some integer $j, P_{j}$ is paracontracting and for infinitely many integers $k, T k=j$.

2) $T^{-1} Q$ is paracontracting with respect to $\|\cdot\|_{X}$, the sequence $\{T k\}_{k=0}^{\infty}$ is unbounded, and either $G^{-1} Q x \gg 0$ or $G^{-1} H x \gg 0$.

The sequence of iterations $\{z k\}_{k=1}^{\infty}$ generated by the scheme given in (7) converges to a solution of the system $A x=b$.

Proof. We have the identity that

$$
I-P_{i}=\sum_{j=0}^{i-1}\left(R_{i}\right)^{j} G^{-1} A
$$

from which it follows that $x$ is a positive vector for which

$$
x \geq P_{1} x \geq P_{2} x \geq \cdots
$$

showing that for each $i \geq 1, P_{i}$ is no expansive with respect to the monotonic vector norm induced by $x$. Also the proof of (2) is clear because the unboundness of the sequence $\{T k\}_{k=0}^{\infty}$ together with the existence of the limit in Equation (10) now means that the sequence of matrices $\left\{P_{T k}\right\}_{k=0}^{\infty}$ contains an infinite subsequence of matrices which converges to the paracontracting matrix V.

\section{Conclusion}

The conditions under which the product $\prod_{i=0}^{\infty} P_{i}$ of matrices converges are explained and we apply the results for the convergence of inner-outer iteration schemes for solving singular consistent linear system of equations.

\section{REFERENCES}

[1] Y. S. Hanna, "On the Solutions of Tridiagonal Linear System," Applied Mathematics and Computation, Vol. 189, 2007, pp. 2011-2016.

[2] R. Bru, L. Elsner and M. Neumann, "Convergence of Infinite Products of Matrices and Inner-Outer Iteration Schemes," Electronic Transactions on Numerical Analysis, Vol. 2, 1994, pp. 183-193.

[3] N. K. Nichols, "On the Convergence of Two-Stage Iterative Processes for Solving Linear Equations," SIAM Journal on Numerical Analysis, Vol. 10, No. 3, 1973, pp. 460-469. doi: $10.1137 / 0710040$

[4] P. J. Lanzkron, D. J. Rose and D. B. Szyld, "Convergence of Nested Classical Iterative Methods for Linear Systems," Numerische Mathematik, Vol. 58, 1991, pp. 658702.

[5] A. Frommer and D. B. Szyld, "H-Splittings and TwoStage Iterative Methods," Numerische Mathematik, Vol. 63, No. 1, 1992, pp. 345-356. doi:10.1007/BF01385865

[6] I. Daubechifs and J. C. Lagarias, "Sets of Matrices All Infinite Products of Which Converge," Linear Algebra and Its Applications, Vol. 161, 1992, pp. 227-263. doi:10.1016/0024-3795(92)90012-Y

[7] R. Bru, L. Elsner and M. Neumann, "Models of Parallel Chaotic Iteration Methods," Linear Algebra and Its Applications, Vol. 102, 1988, pp. 175-192. doi:10.1016/0024-3795(88)90227-3

[8] L. Elsner, I. Koltracht and M. Neumann, "On the Convergence of Asynchronous Paracontractions with Application to Tomographic Reconstruction from Incomplete Data," Linear Algebra and Its Applications, Vol. 130, 1990, pp. 65-82. doi:10.1016/0024-3795(90)90206-R

[9] S. Nelson and M. Neumann, "Generalizations of the Projection Method with Applications to SOR Theory for Hermitian Positive Semidefinite Linear Systems," $\mathrm{Nu}$ merische Mathematik, Vol. 51, No. 2, 1987, pp. 123-141. doi:10.1007/BF01396746

[10] R. S. Varga, "Matrix Iterative Analysis," Prentice-Hall, 
Englewood Cliffs, 1961.

[11] J. M. Optega and W. Rueinboldt, "Monotone Iterations for Nonlinear Equations with Application to Gauss-Seidel Methods," SIAM Journal on Numerical Analysis, Vol. 4, No. 2, 1967, pp. 171-190. doi:10.1137/0704017

[12] M. Neumann and R. J. Plemmons, "Convergent Nonnegative Matrices and Iterative Methods for Consistent
Linear Systems," Numerische Mathematik, Vol. 31, No. 3, 1978, pp. 265-279. doi:10.1007/BF01397879

[13] M. Neumann and R. J. Plemmons, "Generalized Inverse-Positivity and Splittings of M-Matrices," Linear Algebra and Its Applications, Vol. 23, 1979, pp. 21-35. doi:10.1016/0024-3795(79)90090-9 